\title{
New COMPASS Results on Longitudinal Spin Effects
}

\author{
Marcin Stolarski on behalf of the COMPASS Collaboration
}

LIP-Lisboa, Av. Elias Garcia 14-1 1000-149 Lisboa Portugal

\begin{abstract}
.
A short review of the COMPASS results obtained in polarized deep inelastic scattering of polarized muons off longitudinally polarized proton and deuteron targets is given. Two new measurements of the gluon polarization $\Delta G / G$ are discussed. A new LO result from the analysis of high- $p_{T}$ hadron pairs for $Q^{2}>1(\mathrm{GeV} / \mathrm{c})^{2}$ and separated into three experimental points as a function of $x_{G}$ is shown. A new open charm value for $\Delta G / G$ obtained at NLO, and which combines both proton and deuteron data and includes several $D^{0}$ decay channels is presented. Finally results for the LO flavor separation of the three lightest quark polarized parton distributions and charged $\pi$ and $\mathrm{K}$ multiplicities are shown.
\end{abstract}

Keywords: dg/g, dg, gluon polarization, hadron multiplicities, g1p, g1d

PACS: $13.80-\mathrm{r}$

\section{INTRODUCTION}

COMPASS is an experiment at CERN focusing on the spin structure of the nucleon and hadron spectroscopy. Between 2002 and 2004, a $160 \mathrm{GeV} / \mathrm{c}$ polarized muon beam and a two cell polarized ${ }^{6} \mathrm{LiD}$ target were used for spin studies. For the 2006 data taking major upgrades of the spectrometer were made. This included of the RICH detector improvement for particle ID, a new COMPASS solenoid around the target with an angular acceptance increased from 70 mrad to 180 mrad, and a new three cell target. From 2002-2006 the ${ }^{6} \mathrm{LiD}$ target was used as it is optimal for $\Delta G / G$ measurements In 2007 an ammonia $\mathrm{NH}_{3}$ target was used so that spin effects on polarized protons could be studied.

In this paper selected results on longitudinal spin effects measured by COMPASS are presented. The focus is on new $\Delta G / G$ results obtained in the open charm and in High- $p_{T}$ hadron pairs analyses. The LO flavor separation is also discussed with special attention of $\Delta S$ distribution.

\section{GLUON POLARIZATION}

The EMC results [1] in the late 80s suggested that the quarks may be responsible for only a small fraction of the nucleon spin contrary to the naive expectation that the spin of the nucleon may be built from valence quarks only. Several other experiments in world leading laboratories of particles physics (at CERN, DESY, JLAB and SLAC) confirmed the EMC observation with improved precision. The contribution of quarks to the nucleon spin is now believed to be about $30 \%$. More generally the spin of the nucleon can be decomposed as:

$$
S_{z}=\frac{1}{2}=\frac{1}{2} \Delta \Sigma+\Delta G+L_{q}+L_{g},
$$

where $\Delta \Sigma$ and $\Delta G$ are the polarized quark and gluon contributions to the nucleon spin and $L_{q, g}$ are their angular momenta. In COMPASS so far the gluon polarization is measured by studying photon-gluon fusion processes (PGF). This can be done in $i$ ) open charm studies and $i i$ ) in the analysis of hadrons produced with high transverse momenta (HipT). In the former case the analysis is free from any physical background. It is believed that the charm quarks are not constituents of the nucleon and so the appearance of the charm mesons in the final state suggest that a PGF process took place. Unfortunately due to the large charm mass, in the COMPASS energy range the production cross section for charm mesons is small, and also branching ratio for the $D^{0} \rightarrow K \pi$ is below $4 \%$. Therefore the analysis has limited statistical precision. The latter method has an advantage over open charm that the production cross-section is larger therefore the statistical error of $\Delta G / G$ will be reduced. On the other hand the HipT analysis is not background free. Almost all the crucial ingredients for this analysis have to be obtained from MC simulation using generators like LEPTO. Therefore in this analysis the systematic error plays a very important role. 


\section{Open charm analysis}

In the current open charm analysis all available data from 2002-2007 were used. In order to improve the statistical error of $\Delta G / G$ five different $D$ mesons decay modes are studied. In total there are about $65000 D^{0}$ candidates and about $29000 D^{*}$ candidates out of which 13000 are in the golden channel $D^{*} \rightarrow K \pi \pi_{\text {slow }}$. In a simplified approach the gluon polarization $\Delta G / G$ can be obtained from:

$$
\frac{\Delta G}{G}=\frac{1}{P_{T} P_{b} f a_{L L} \frac{S}{S+B}} A^{\mu N \rightarrow D^{0}+X}
$$

where $P_{T, b}$ are the target and beam polarization, respectively, $f$ is the dilution factor of the material which takes into account the fraction of polarizable nucleons in the target, including radiative corrections. $a_{L L}$ is the so called analysing power which represents the polarization transfer from muon to photon and from gluon to charm quarks. The $\frac{S}{S+B}$ is the ratio between signal and signal plus combinatorial background, and finally the $A^{\mu N \rightarrow D^{0}+X}$ is the measured asymmetry. The method which is actually used in the analysis is much more complex $e$.g. it allows the simultaneous extraction of signal and background asymmetries, details can be found in [2]. To increase the statistical significance of the results the events are weighted on an event by event basis. The weight for each event is $\frac{1}{P_{T} P_{b} f a_{L L}} \frac{S}{S+B}$. In the current analysis $S /(S+B)$ is parametrized using a Neural Network approach. As a model of background the wrong charge combinations (wcc) are used e.g. $K^{+} \pi^{+}$, while the $D^{0}$ decays into $K^{+} \pi^{-}$a good charge combination $(g c c)$. The NN is trying to find the differences between $w c c$ and $g c c$ in the phase space of the input parameters which can be attributed to the $D^{0}$ mesons present in the good charge combination sample.

The final result $\Delta G / G=-0.08 \pm 0.21 \pm 0.11$ is compatible with zero and the error bar is reduced by a factor of 1.3 w.r.t. the previously published value [2]. The current value is about 0.4 higher than previously published. With the additional years and new channels included in the analysis, and taking into account the correlation between the two analyses, the resulting change corresponds to a bit more than two sigma. We treat it as a statistical fluctuation.

The gluon polarization in NLO approximation, based on [3], was recently extracted in COMPASS. The AROMA generator is used with active parton shower option. The parton shower simulates the phase-space for NLO correction, which can be calculated on the event by event basis. Large differences are observed between $a_{L L}$ and $x_{G}$ for LO and NLO order $c$.f. Fig. 1. In addition, in NLO part of the $D^{0}$ doesn't come from PGF process, but is sensitive to inclusive spin asymmetries $A_{1}^{p, d}$. This correction was found to be small, but it is included in the analysis. The final results of the NLO analysis is $\Delta G / G_{N L O}=-0.20 \pm 0.21 \pm 0.08$.

In addition unpolarized production of $D^{*}$ mesons was studied. The preliminary measured visible cross-section in the range of $D^{*}$ energies $22-86 \mathrm{GeV}$ was found to be $1.9 \pm 0.4 \mathrm{nb}$, in agreement with AROMA generator which gives $2.6 \mathrm{nb}$. The shape of $D^{*}$ differential cross-section as a function of kinematic variables is also well described by the AROMA generator. However, large non-zero asymmetries are observed between the cross-section of $D^{*+}$ and $D^{*-}$. This may suggest that additional non-PGF processes may contribute to the $D^{*}$ production. An example of the crosssection asymmetries as a function of virtual photon energy and a ratio of $D^{0}$ energy to the photon energy is presented in Fig. 2.
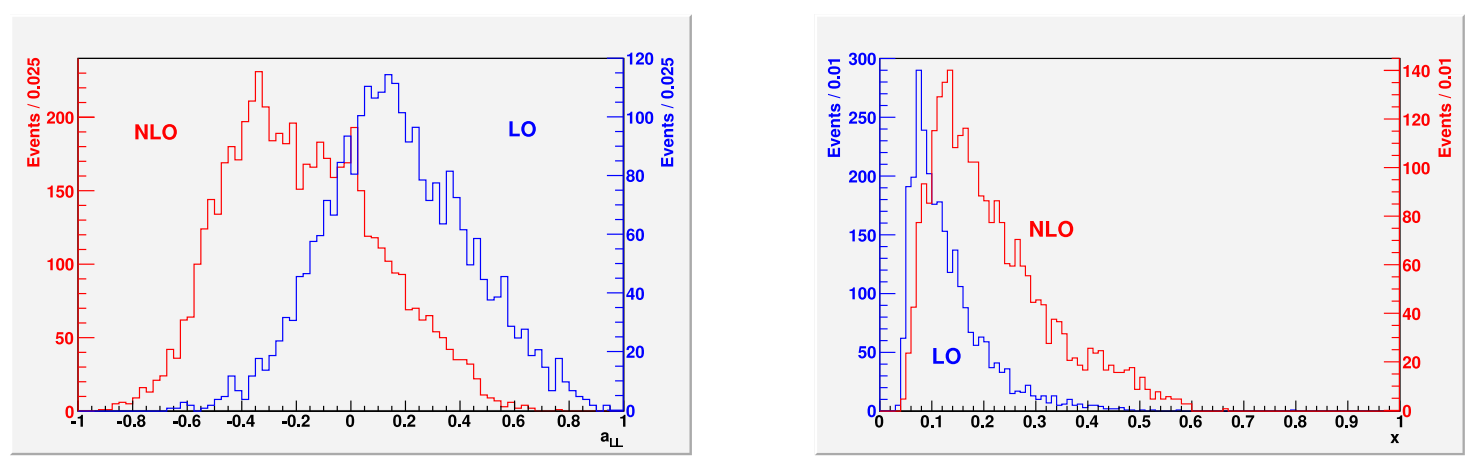

FIGURE 1. The $a_{L L}$ and $x_{G}$ comparison between LO and NLO for open charm analysis. 

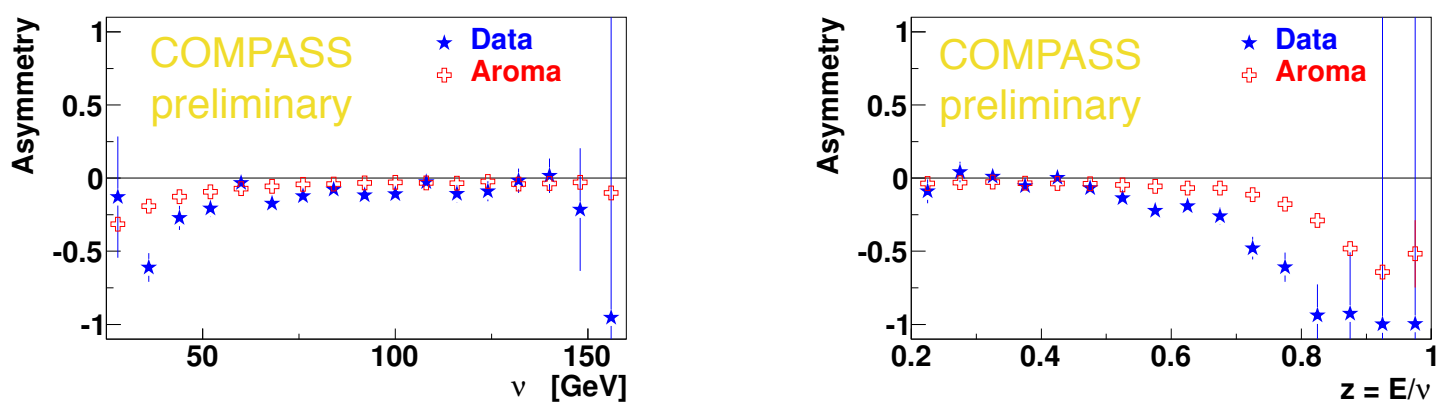

FIGURE 2. The cross-section asymmetries between $D^{*+}$ and $D^{*-}$ as a function of virtual photon energy (left) and ratio of $D^{0}$ and virtual photon energy (right).

\section{High- $p_{T}$ hadron pair analysis}

The results of $\Delta G / G$ analysis form $Q^{2}>1(\mathrm{GeV} / \mathrm{c})^{2}$ high- $p_{T}$ hadron pairs are presented. Data come from 20022006 years when COMPASS used LiD target. As the $Q^{2}$ ensures perturbative scale the cut on hadron $p_{T}$ can be keep low, here $p_{T, 1}=0.7$ and $p_{T, 2}=0.4 \mathrm{GeV} / \mathrm{c}$, for the first and the second hadron respectively. The total number of events in the selected sample is about 7.3 millions. The sample is strongly contaminated by a non-PGF processes. This background is related to Leading Process (LP) i.e. photon-quark scattering and QCD Compton process (QCDC), where the scattered quark emits in addition a gluon. It is expected that for higher $p_{T}$ of hadrons the PGF fraction will grow while LP decreases. The observed asymmetry in a two hadron sample can be written as:

$$
A_{L L}^{2 h}\left(x_{B j}\right)=R_{P G F} a_{L L}^{P G F} \frac{\Delta G}{G}\left(x_{G}\right)+R_{L P} D A_{1}^{L O}\left(x_{B j}\right)+R_{Q C D C} a_{L L}^{Q C D C} A_{1}^{L O}\left(x_{C}\right)
$$

where $A_{1}^{L O} \equiv \frac{\sum_{i} e_{i}^{2} \Delta q_{i}}{\sum_{i} e_{i}^{2} q_{i}}, R \mathrm{~s}$ are fractions of the sub-processes (LO, PGF, QCDC) and $a_{L L} \mathrm{~s}$ - analyzing powers for PGF and QCDC. Unfortunately $R \mathrm{~s}$ and $a_{L L} \mathrm{~s}$ cannot be determined from data they have to be taken from elsewhere e.g. MC simulation. Therefore a good agreement between data and $\mathrm{MC}$ is crucial for this analysis. In the above equation there are two unknowns $\Delta G / G$ and $A_{1}^{L O}$, to be obtain $\Delta G / G$ additional information is needed. This information is taken from the inclusive sample, where inclusive asymmetry is described in a similar way as the asymmetry of two hadron sample

$$
\begin{gathered}
A_{1}^{d}\left(x_{B j}\right)=R_{P G F}^{i n c l} a_{L L}^{i n c l, P G F} \frac{\Delta G}{G}\left(x_{G}\right)+R_{L P}^{i n c l} D A_{1}^{L O}\left(x_{B j}\right)+R_{Q C D C}^{i n c l} a_{L L}^{i n c l, Q C D C} A_{1}^{L O}\left(x_{C}\right) \\
\Delta G / G=\Delta G / G\left(x_{G}^{a v}\right)=\frac{A_{L L}^{2 h}\left(x_{B j}\right)+A^{\text {corr }}}{\beta}
\end{gathered}
$$

where $\beta=a_{L L}^{P G F} R_{P G F}-a_{L L}^{P G F, i n c l} R_{P G F}^{\text {incl }}\left(\frac{R_{L}}{R_{L}^{\text {incl }}}+\frac{R_{C}}{R_{L}^{\text {incl }}} \frac{a_{L L}^{C}}{D}\right)$ and $A^{\text {corr }}$ is a function of $A_{1}^{d}\left(x_{B j} \sim 0.03\right)$ and $A_{1}^{d}\left(x_{C} \sim 0.11\right)$. Observe that $A_{1}^{d}\left(x_{C} \sim 0.11\right)$ is non zero and $A^{c o r r}$ plays an important role in $\Delta G / G$ extraction. To reduce statistical error of $\Delta G / G$ the weighted method of the asymmetry extraction is used. All $R \mathrm{~s}$ and $a_{L L} \mathrm{~s}$ have to be known on the event-by-event basis. We use a Neural Network trained on MC to obtain parametrizations of $R s$ and $a_{L L} \mathrm{~s}$. The LEPTO generator is used with parton shower on, and MSTW08LO as a source of parton distribution functions. To improve data/MC agreement the intrinsic $k_{T}$ of quarks inside nucleon and fragmentation parameters were adjusted. Example of parametrization obtained from $\mathrm{NN}$ and data/MC agreement for selected hadron variables is presented in Fig. 3 The three first plots shows comparison between fraction of the processes LP, QCDC and PGF observed in MC and parametrized by the NN as a function of $\sum p_{T}^{2}$. Good agreement between them is observed. In the last three plots example of data/MC ratio is shown for $p_{L, 1}, p_{T, 1}$ and hadron multiplicities for default and COMPASS tunings. Clear improvement is seen for the COMPASS tuning.

COMPASS preliminary results of $\Delta G / G$ extracted in this analysis is $\Delta G / G=0.125 \pm 0.060 \pm 0.065$. The major contribution to the systematic error (0.045) comes from MC. In addition COMPASS for the first time obtained results in three bins of $x_{G}$. Within statistical errors these three results agree with each other. The obtained in this analysis results suggest that $\Delta G / G$ is small $c . f$. Fig. 4 . 

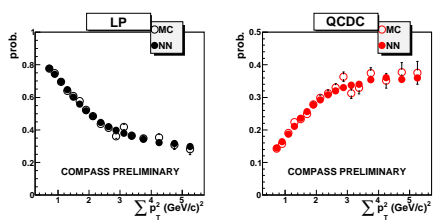

QCDC
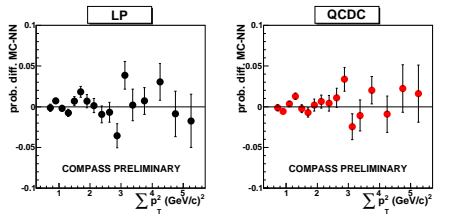

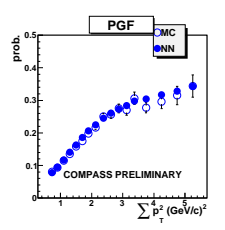

PGF

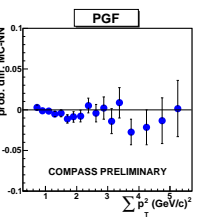

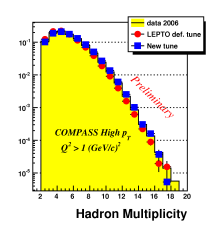

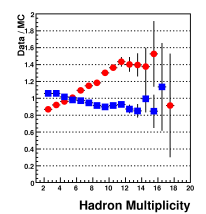

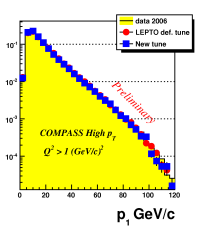

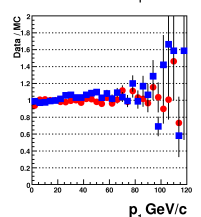

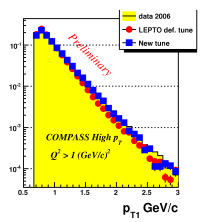

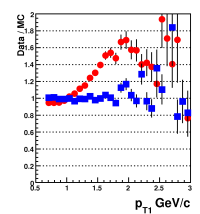

FIGURE 3. The example of a NN parametrization of the processes fractions (3 left plots) and data/MC agreement for different LEPTO tunings for hadron multiplicities, longnitudinal and transverse momentum of the first hadron.

COMPASS also obtained $\Delta G / G$ from the $\mathrm{Q}^{2}<1(\mathrm{GeV} / \mathrm{c})^{2}$ two hadron sample. Here cuts on hadron $p_{T}$ must be kept high in order to ensure perturbative regime. The preliminary result is $\Delta G / G=0.016 \pm 0.058 \pm 0.014 \pm 0.052 \pm 0.013$.

The results of COMPASS $\Delta G / G$ analyses are summarized in Fig. 4. In all analyses extracted $\Delta G / G$ is small and consistent with zero. The results agree well with each other as well as with measurements from SMC and HERMES experiments.
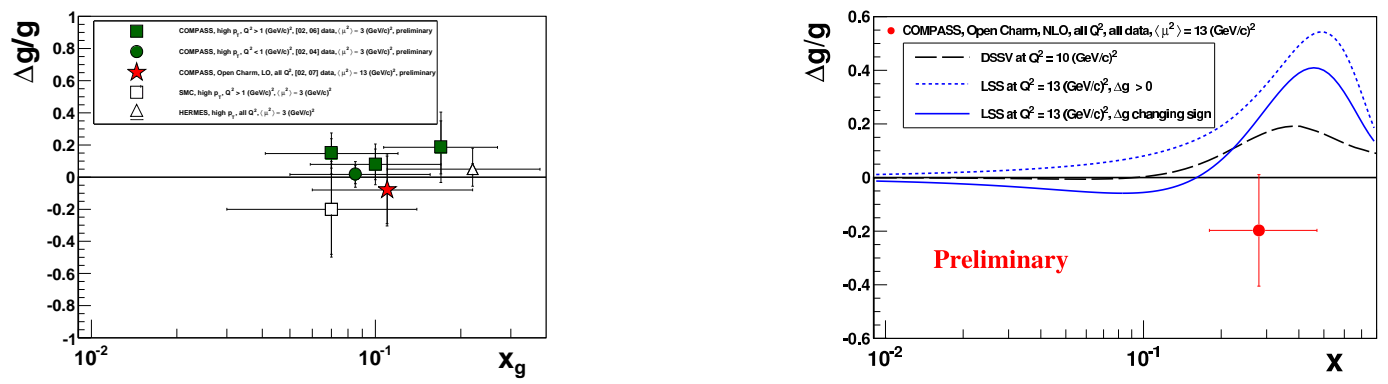

FIGURE 4. Summary of $\Delta G / G$ results obtained in LO and NLO analysis.

Currently the NLO analysis of a single high- $p_{T}$ hadron is ongoing for $Q^{2}<0.1(\mathrm{GeV} / \mathrm{c})^{2}$ events. In the first step hadron cross-section as a function of $p_{T}$ was compared with theoretical prediction [4]. A good agreement is observed for the sum of $h^{+}$and $h^{-}$, but unexpectedly for $p_{T}$ as low as $1 \mathrm{GeV} / \mathrm{c}$ there is about $15 \%$ more $h^{+}$than $h^{-}, c . f$ Fig. 5. As in COMPASS for $Q^{2}<0.1$ average $x_{B j}$ is well below 0.005 , there is no valence quarks which could justify so strong charge asymmetry.
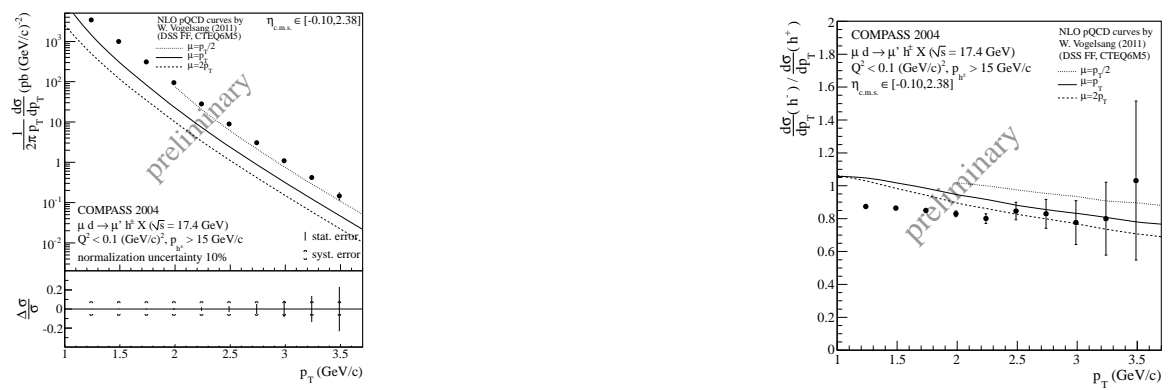

FIGURE 5. Single high- $p_{T}$ hadron analysis $Q^{2}<0.1(\mathrm{GeV} / \mathrm{c})^{2}$, observed cross-section of hadron production as a function of $p_{T}$ (left); cross-section ratio $h^{-} / h^{+}$(right). 


\section{FLAVOR SEPARATION}

COMPASS is also analysing the semi-inclusive asymmetries for kaons and pions on both proton and deuteron targets. In the LO approximation the hadron asymmetry can be expressed as

$$
A_{1}^{h}\left(x, Q^{2}, z\right)=A_{1}^{h}\left(x, Q^{2}, z\right)=\frac{\sum_{q} e_{q}^{2} \Delta q\left(x, Q^{2}\right) D_{q}\left(z, Q^{2}\right)}{\sum_{q} e_{q}^{2} q\left(x, Q^{2}\right)}
$$

where $e_{q}$ is quark electric charge, $(\Delta) q$ (polarized) parton distribution function and $D_{q}$ are fragmentation functions (FF). With inclusive and semi-inclusive asymmetries one have 10 measured asymmetries and 5 unknown parameters $(\Delta u, \Delta d, \Delta \bar{u}, \Delta \bar{d}, \Delta s)$, The flavor separation of quark helicity distributions is possible using just a linear algebra. Some results were already published in [5] using FF from DSS parametrization [6]. The results are summarized in Fig. 6 (left). In general good agreement is observed between COMPASS results and DSSV parametrization [7].

From the inclusive asymmetries it is known that the strange sea polarization is negative, $\Delta S=\int_{0}^{1}(\Delta s(x)+\Delta \bar{s}) d x=$ $-0.09 \pm 0.01 \pm 0.02$.. This tendency is so far not observed in COMPASS and HERMES semi-inclusive analyses. However, as pointed out in [5] the $\Delta S$ obtained in semi-inclusive analysis strongly depends upon the choice of the fragmentation functions used. COMPASS try to extract these FF from data alone. One way of extracting them is to study hadron multiplicities i.e. number of hadrons produced per DIS event. So far charged $K$ and $\pi$ multiplicities are available, they are shown in Fig. 6 (right). A good agreement between DSS parametrization and data is observed for $\pi$ but clear discrepancies are seen for the $K$ case. The extraction of FF from these multiplicities is in progress.
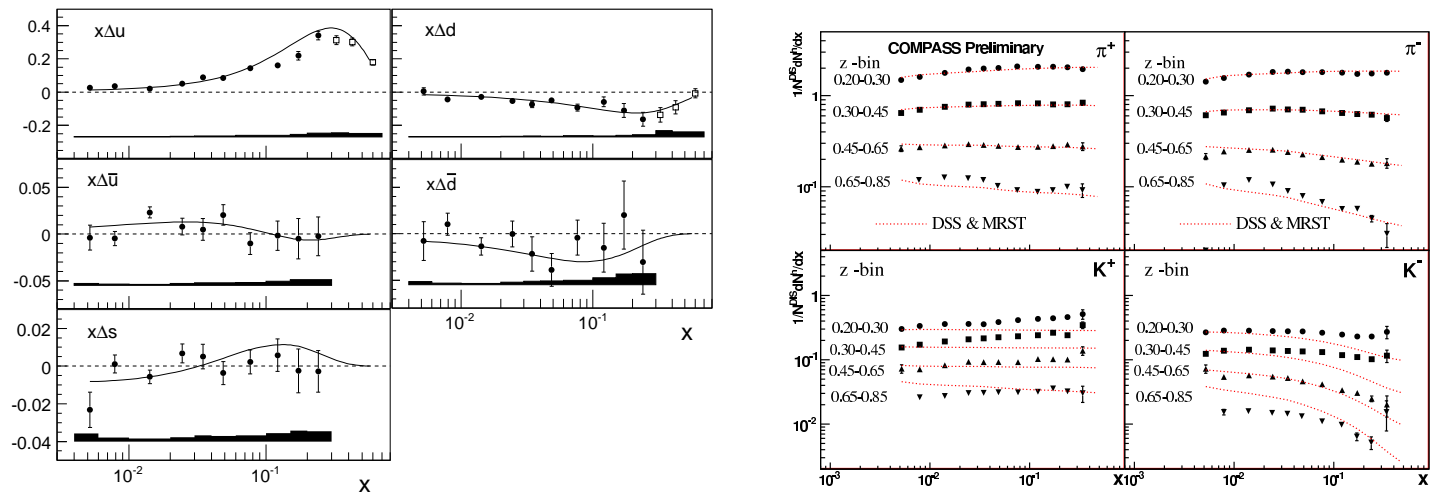

FIGURE 6. COMPASS results of LO flavor separation of polarized quark helicity distributions and comparison with DSSV NLO parametrization (left); Multiplicities of charged $\pi$ and $K$ measured in COMPASS and expectation from DSS parametrization (right).

\section{ACKNOWLEDGMENTS}

This research was supported by the Portuguese Fundação para a Ciência e a Tecnolagia

\section{REFERENCES}

1. EMC, J. Ashman et al., Nucl. Phys. B 328,1 (1988); Phys. Lett. B 206, 364 (1988).

2. COMPASS, M. Alekseev et al., Phys. Lett. B 676, 31 (2009).

3. I. Bojak, M. Stratmann, Nucl. Phys. B 540, 345 (1999).

4. B. Jeager, M. Stratmann and V. Vogelsang, Phys. J. C 44, 533 (2005).

5. COMPASS, M. Alekseev et al., Phys. Lett. B 693, 227, (2009).

6. D. de Florian, R. Sassot, M. Stratmann, Phys. Rev. D 75, 114010 (2007).

7. D. de Florian, R. Sassot, M. Stratmann, W. Vogelsang, Phys. Rev. Lett. 101, 072001 (2008); Phys. Rev. D 80, 034030 (2009). 